\title{
Time Variability and Simmons-Wallace-Branstator Instability in a Simple Nonlinear One-Layer Model
}

\author{
LORENZO M. Polvani \\ Department of Applied Physics and Applied Mathematics, Columbia University, New York, New York \\ J. Gavin Esler and R. Alan Plumb \\ Program in Atmospheres, Ocean and Climate, Massachusetts Institute of Technology, Cambridge, Massachusetts
}

(Manuscript received 20 January 1998, in final form 26 May 1998)

\begin{abstract}
Using a global, one-layer shallow water model, the response of a westerly flow to a localized mountain is investigated. A steady, linear response at small mountain heights successively gives way first to a steady flow in which nonlinearities are important and then to unsteady, but periodic, flow at larger mountain heights. At first the unsteady behavior consists of a low-frequency oscillation of the entire Northern Hemisphere zonal flow. As the mountain height is increased further, however, the oscillatory behavior becomes localized in the diffluent jet exit region downstream of the mountain. The oscillation then takes the form of a relatively rapid vortex shedding event, followed by a gradual readjustment of the split jet structure in the diffluent region. Although relatively simple, the model exhibits a surprisingly high sensitivity to slight parameter changes. A linear stability analysis of the time-averaged flow is able to capture the transition from steady to time-dependent behavior, but fails to capture the transition between the two distinct regimes of time-dependent response. Moreover, the most unstable modes of the time-averaged flow are found to be stationary and fail to capture the salient features of the EOFs of the full time-dependent flow. These results therefore suggest that, even in the simplest cases, such as the one studied here, a linear analysis of the time-averaged flow can be highly inadequate in describing the full nonlinear behavior.
\end{abstract}

\section{Introduction}

Since the work of Simmons, Wallace, and Branstator (1983; hereafter SWB), the linear instability of the zonally asymmetric, time-averaged flow in the upper troposphere has been suggested as an important ingredient in the maintenance of low frequency variability, even if its precise role has not been fully clarified. SWB and subsequent work (Frederiksen and Webster 1988; Borges and Sardeshmukh 1995; Branstator and Held 1995; Huang and Robinson 1995) identified the instability as being localized in the vicinity of the upper-tropospheric jet exit, and found growing disturbances that were either

\footnotetext{
${ }^{1}$ We refer to this instability as the "Simmons-Wallace-Branstator" instability.

Corresponding author address: Dr. Lorenzo M. Polvani, Department of Applied Physics and Applied Mathematics, Columbia University, Seeley W. Mudd Bldg., Rm. 209, New York, NY 10027. E-mail: polvani@columbia.edu
}

stationary or slowly propagating and whose amplitudes are concentrated near the jet exit region.

In most of the previous studies, the basic state was taken to be an observed, time-averaged upper-tropospheric flow [although Huang and Robinson (1995) took a simplified representation], and the characteristics of growing disturbances were investigated, assuming the background flow to be maintained by external, fixed, and ad hoc forcing that does not interact with the growing disturbances. Andrews (1984) has shown that, in fact, the nature of the instability may be sensitive to assumptions made about this external forcing. What remains unclear, moreover, is the detailed dynamics of the instability - in what way, for example, the instability depends on the degree of nonzonality in the basic flow, and how the equilibrated, nonlinear, oscillations of the full flow relate to the growing disturbances calculated from linearization about the time-averaged state.

In this paper we take a different approach. Rather than attempting to describe the linear instability characteristics of observed flows, we construct a simple model to study how a one-layer westerly flow responds to the presence of a localized forcing. Although we explicitly use topographic forcing in this study, we do 
TABLE 1. A summary of the parameter settings used in the experiments.

\begin{tabular}{rrr}
\hline \hline$A_{F}(\mathrm{~m})$ & Resolution & $v\left(\mathrm{~m}^{6} \mathrm{~s}^{-1}\right)$ \\
\hline 100 & $\mathrm{~T} 42$ & $6 \times 10^{26}$ \\
500 & $\mathrm{~T} 42$ & $6 \times 10^{26}$ \\
1000 & $\mathrm{~T} 42$ & $6 \times 10^{26}$ \\
1500 & $\mathrm{~T} 42$ & $6 \times 10^{26}$ \\
2000 & $\mathrm{~T} 42$ & $6 \times 10^{26}$ \\
2500 & $\mathrm{~T} 42$ & $6 \times 10^{26}$ \\
3000 & $\mathrm{~T} 42$ & $6 \times 10^{26}$ \\
3500 & $\mathrm{~T} 42$ & $6 \times 10^{26}$ \\
4000 & $\mathrm{~T} 42$ & $6 \times 10^{26}$ \\
4500 & $\mathrm{~T} 42$ & $1 \times 10^{27}$ \\
5000 & $\mathrm{~T} 42$ & $3.6 \times 10^{27}$ \\
5000 & $\mathrm{~T} 21$ & $2 \times 10^{28}$ \\
5000 & $\mathrm{~T} 42$ & $5 \times 10^{25}$ \\
5000 & $\mathrm{~T} 85$ &
\end{tabular}

so with the intention that this be a proxy for any localized stationary wave generator. Furthermore, unlike most previous work, we explicitly avoid using an ad hoc forcing term to maintain a prescribed time-averaged flow. We simply observe and report how the response changes as the forcing amplitude is increased and then compare the features of the linear instabilities of the time-averaged flow with those of the fully nonlinear time-dependent flow.

\section{The model}

The physical model used in this paper is an incompressible, one-layer, spherical fluid shell of mean depth $10 \mathrm{~km}$, obeying the shallow water equations:

$$
\begin{aligned}
& D_{t} \mathbf{v}=-f \mathbf{k} \times \mathbf{v}-g \boldsymbol{\nabla}\left(h+h_{F}\right)-\tau_{D}^{-1}\left(\mathbf{v}-\mathbf{v}_{0}\right) \\
& D_{t} h=-h \boldsymbol{\nabla} \cdot \mathbf{v},
\end{aligned}
$$

where $h$ is the thickness of the fluid layer, $\mathbf{v}=(u, v)$ is the two-dimensional velocity vector, $f$ is the Coriolis parameter, $g$ is the gravitational acceleration, $\tau_{D}$ is a drag coefficient, and $D_{t} \equiv \partial_{t}+\mathbf{v} \cdot \boldsymbol{\nabla}$ is the material derivative.

These equations are numerically integrated using a standard pseudospectral method (Hack and Jacob 1992). Unless otherwise specified, the computations reported below are performed using a T42 truncation (corresponding to a Gaussian grid with approximately $2.8^{\circ}$ grid size and a standard $\nabla^{6}$ hyperdissipation on the left side of (1). The hyperdiffusivity $\nu$ is adjusted to the lowest possible value consistent with numerical stability in each experiment (see Table 1). As a guide, the value $6 \times 10^{26} \mathrm{~m}^{6} \mathrm{~s}^{-1}$ corresponds approximately to a $5-\mathrm{h}$ $e$-folding scale for the smallest resolved scale $n=42$. The dependence of the model response on the parameter $\nu$ is discussed below in section 4.

The flow is initialized with a latitude-dependent zonal velocity $\mathbf{v}_{0}$ given by

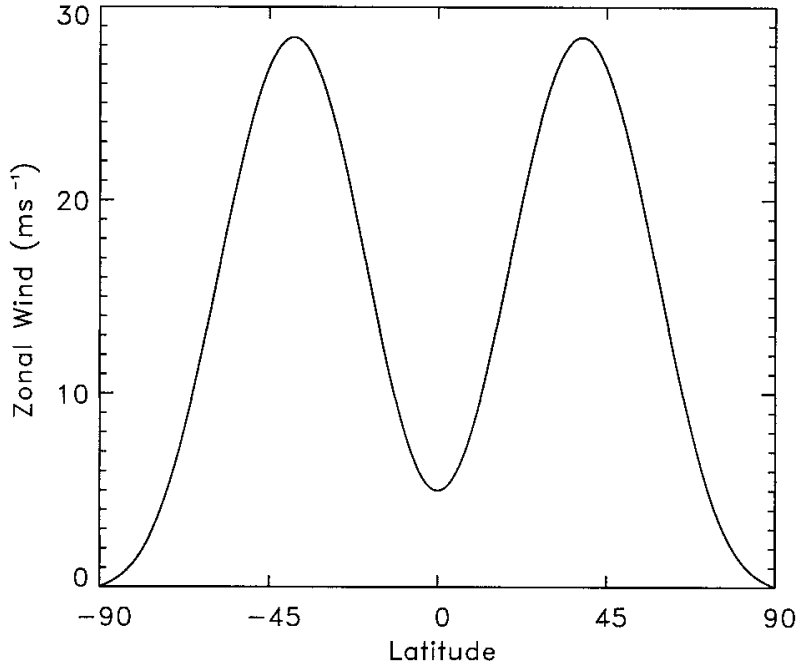

FIG. 1. The zonal flow $u_{0}(\phi)$ used to initialize and drag the model.

$$
\begin{aligned}
\mathbf{v}(\varphi, t=0) & =\mathbf{v}_{0}=\left(u_{0}, v_{0}\right) \\
& =\left(\alpha_{1} \cos \varphi+4 \alpha_{2} \sin ^{2} \varphi \cos ^{3} \varphi, 0\right),
\end{aligned}
$$

where $\varphi$ is the latitude. With the choice $\alpha_{1}=5 \mathrm{~m} \mathrm{~s}^{-1}$ and $\alpha_{2}=33 \mathrm{~m} \mathrm{~s}^{-1}$, this flow (shown in Fig. 1) is meant to be representative of the upper troposphere during the Northern Hemisphere winter. For later reference we note that this zonal flow is barotropically stable, in the sense that the potential vorticity gradient never changes sign. Note also that the winds at the equator are weak westerlies. The thickness field $h$ is initialized to be in exact nonlinear balance with this wind.

This initially steady zonal balanced flow is then forced using the topographic term $g \nabla h_{F}$ in (1). For simplicity, we choose the topography to be a circular Gaussian mountain (cf. Waugh et al. 1994) given by

$$
h_{F}(\varphi, \lambda, t)=A_{F} T(t) e^{\left(\vartheta / \vartheta_{F}\right)^{2}},
$$

where $\varphi$ and $\lambda$ are latitude and longitude, respectively, and $\vartheta$ is the solid angle between the peak of the topography, located at $\left(\varphi_{F}, \lambda_{F}\right)$ and the point $(\varphi, \lambda)$. In this paper we chose the forcing to be in the middle latitudes $\left(\varphi_{F}, \lambda_{F}\right)=\left(40^{\circ} \mathrm{N}, 90^{\circ} \mathrm{E}\right)$ and set the angular width of the mountain to be $\vartheta_{F}=10^{\circ}$. The function $T(t)=\left(1-e^{-t / \tau_{F}}\right)$ is used to grow the mountain in a smooth way with an $e$-folding time $\tau_{F}=5$ days.

The last term in the momentum equation of (1) is a simple linear drag. Unless otherwise specified, we have used $\tau_{D}=45$ days, and the dependence of the results on this parameter will be discussed in section 4 .

\section{The response}

The key parameter controlling the behavior of the response is the peak mountain height, $A_{F}$, which specifies the amplitude of the forcing. Table 1 shows the 

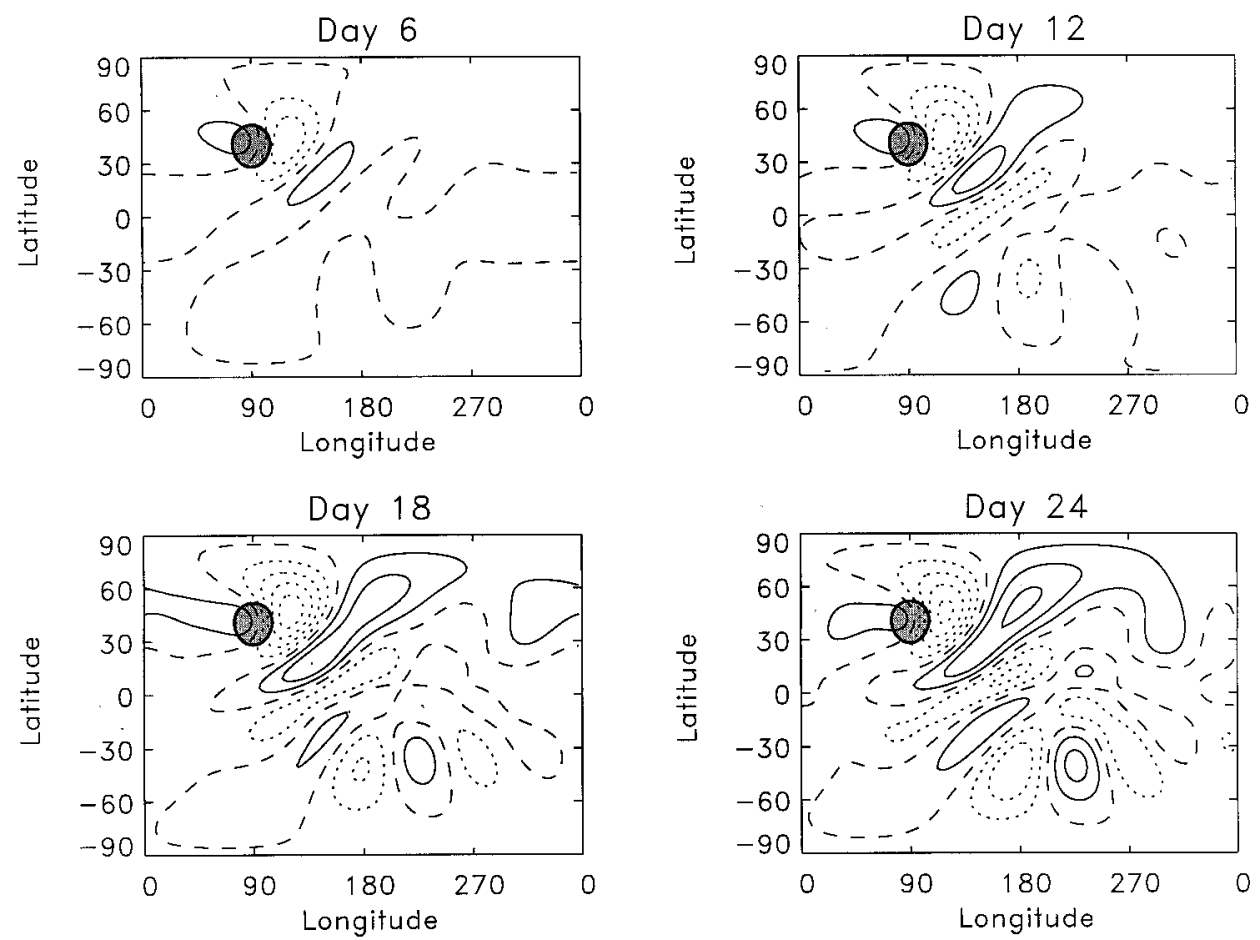

FIG. 2. The perturbation streamfunction for forcing amplitude $A_{F}=500 \mathrm{~m}$ (with respect to the initial state). The contour interval is $1 \times 10^{6} \mathrm{~m}^{2} \mathrm{~s}^{-1}$. The dark shaded region indicates the position of the topography.
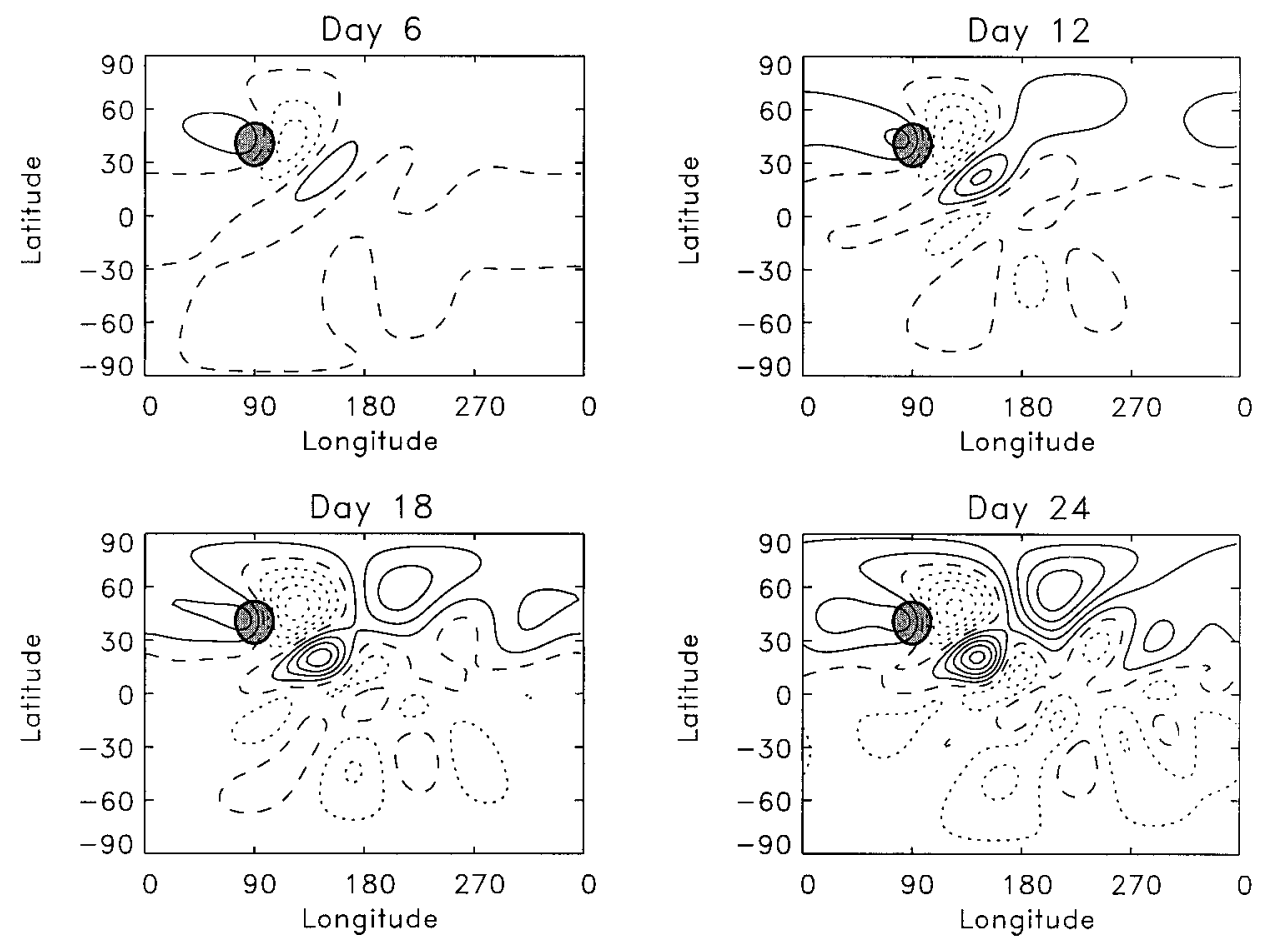

FIG. 3. As in Fig. 2 except for $A_{F}=2000 \mathrm{~m}$. The contour interval is $4 \times 10^{6} \mathrm{~m}^{2} \mathrm{~s}^{-1}$. 

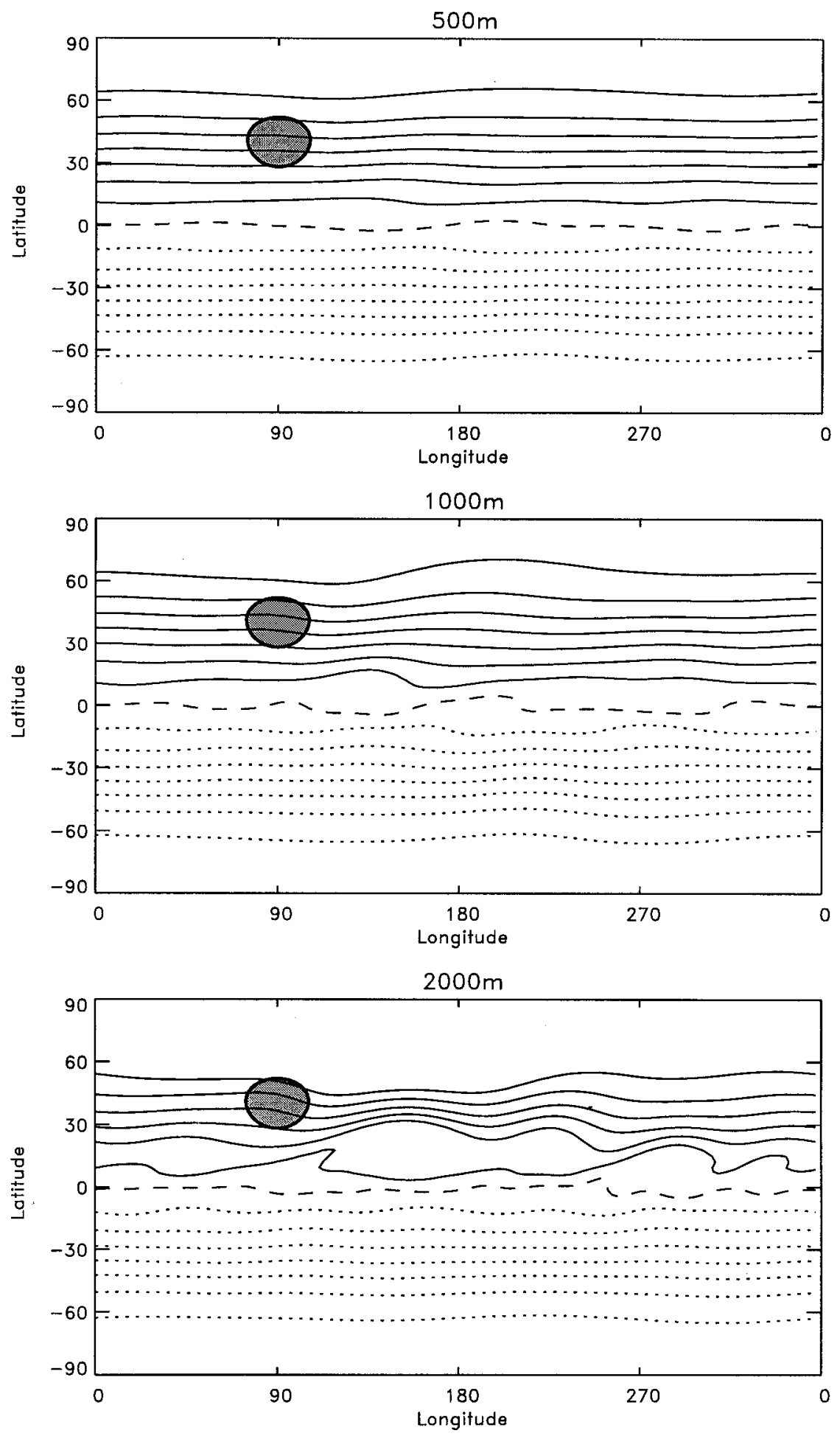

FIG. 4. The PV at day 1000 in the $A_{F}=500,1000$, and $2000 \mathrm{~m}$ experiments. 


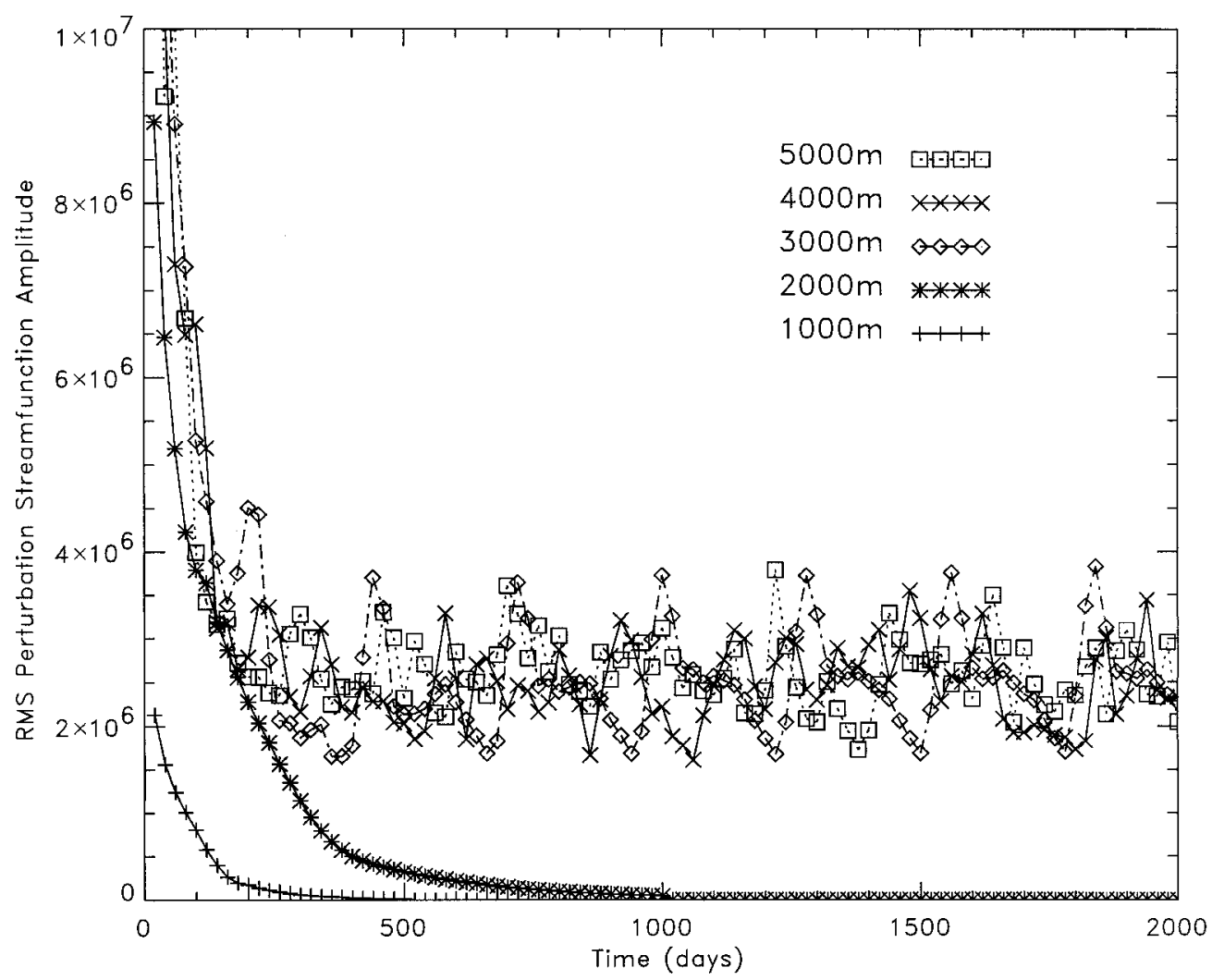

FIG. 5. The evolution of the globally averaged rms perturbation streamfunction amplitude $\sqrt{\left\langle(\psi-\bar{\psi})^{2}\right\rangle}$ for each of the experiments $A_{F}=1000 \mathrm{~m}$ to $5000 \mathrm{~m}$ over the first 2000 model days. For each experiment $\bar{\psi}$ is calculated by time averaging the streamfunction evaluated between day 1500 and 2000 .

range of mountain heights used in the different experiments, along with the corresponding values of the hyperdiffusivity $\nu$. As we will now describe, the flow changes in dramatic ways as the forcing amplitude is varied.

\section{a. Steady response}

For sufficiently small forcing amplitudes (i.e., $A_{F}<$ $1000 \mathrm{~m}$ ) the response is almost entirely linear and the flow rapidly becomes steady. The case with $A_{F}=500$ $\mathrm{m}$ is illustrated in Fig. 2, where the perturbation streamfunction is plotted for the first 24 days. Familiar wave trains are clearly apparent and, in the presence of equatorial westerlies (cf. Fig. 1), they extend into the Southern Hemisphere (Grose and Hoskins 1979; Webster and Holton 1982).

As the forcing amplitude $A_{F}$ is increased the response becomes nonlinear, and the wave propagation into the Southern Hemisphere is hindered by the presence of a subtropical surf zone (Waugh et al. 1994), which acts as a reflector and channels the wave trains along the northern midlatitudes (Brunet and Haynes 1996). Figure 3 illustrates the suppression of the response in the Southern Hemisphere for the case $A_{F}=2000 \mathrm{~m}$.
Up to this amplitude however the flow, whether linear or nonlinear, eventually settles into a steady pattern. The potential vorticity (PV) of the flow at day 1000 (by which time all time variation has vanished) is shown in Fig. 4 as a function of $A_{F}$. Although the flow has become stationary, it bears the marks of the wave breaking for the larger-amplitude cases. The width of the breaking region increases with the forcing amplitude, as in Waugh et al. (1994). Moreover, the steady flow exhibits a region of tight PV gradients just downstream from the forcing, reminiscent of an upper tropospheric jet. As the amplitude of the forcing is increased, not only does the stationary flow exhibit a progressively stronger nonzonal character, but the PV gradients across the jet also become tighter.

\section{b. Unsteady response}

As the forcing amplitude is increased beyond $A_{F}=$ $2000 \mathrm{~m}$, the character of the response again changes dramatically: the long-term response becomes time dependent. This is illustrated in Fig. 5, which shows 2000 days of evolution of the globally averaged root-meansquare (rms) perturbation streamfunction in each of the experiments $A_{F}=1000 \mathrm{~m}, \ldots, 5000 \mathrm{~m}$. The 1000- and 

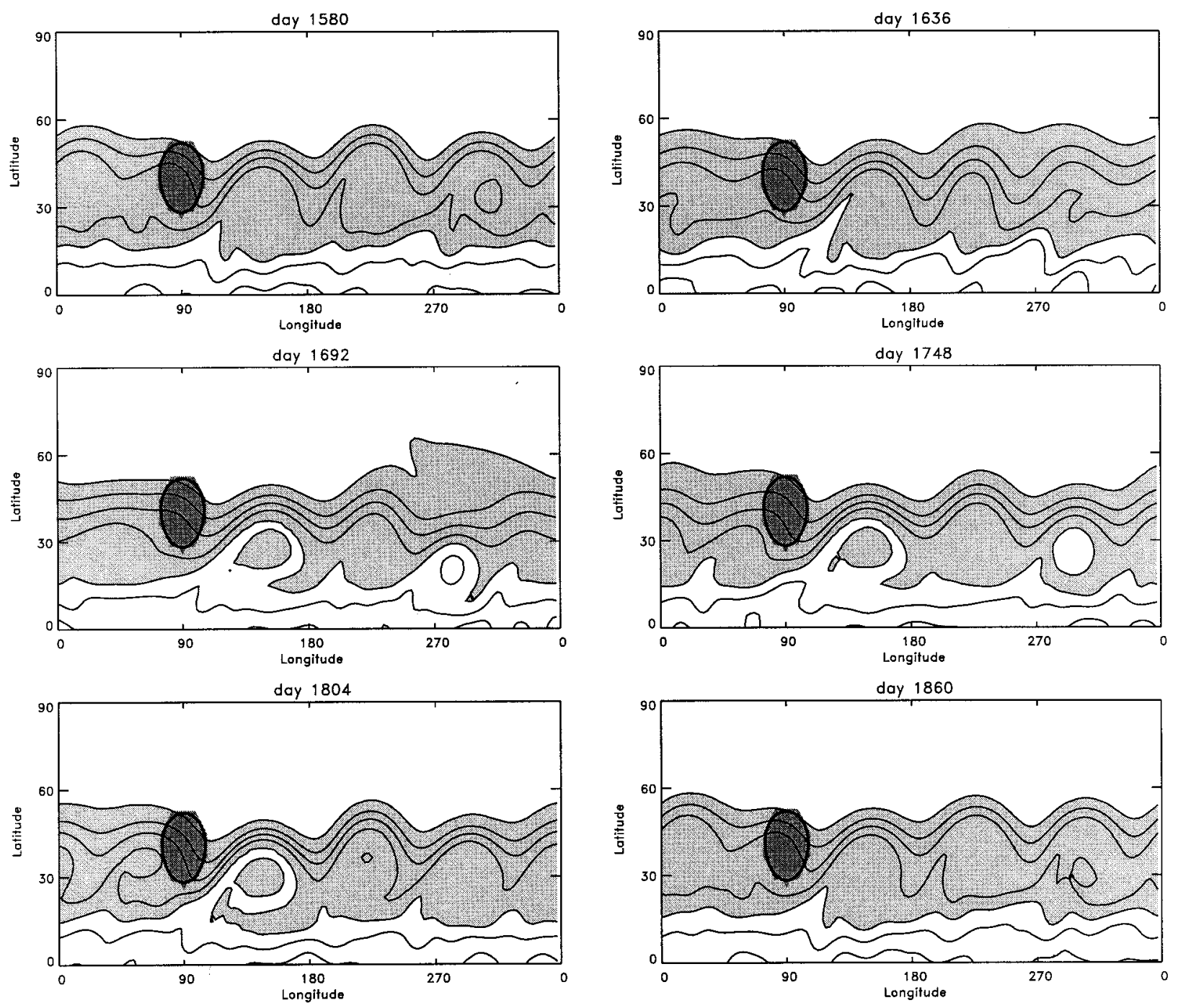

FIG. 6. The Northern Hemisphere PV field over the course of an oscillation in the $A_{F}=3000 \mathrm{~m}$ experiment. The contour interval is $2 \times$ $10^{-9} \mathrm{~m}^{-1} \mathrm{~s}^{-1}$. Values between $4 \times 10^{-9} \mathrm{~m}^{-1} \mathrm{~s}^{-1}$ and $1.2 \times 10^{-8} \mathrm{~m}^{-1} \mathrm{~s}^{-1}$ are lightly shaded. The topography is indicated by the dark shaded region.

2000-m experiments both converge toward steady solutions, but for larger forcing the streamfunction variability asymptotes to an approximately constant value, which is roughly independent of forcing amplitude.

A valuable tool to quantify and describe this variability is complex empirical orthogonal function (EOF) analysis. For a thorough review of this procedure see, for example, Horel (1984). For present purposes, suffice it to say that this analysis makes possible the expression of any evolving quantity (in our case the streamfunction) in terms of its time average and a series of orthogonal complex eigenfunctions,

$$
\psi(x, y, t)=\bar{\psi}(x, y)+\operatorname{Re} \sum_{j} f^{j}(t) \Psi_{j}(x, y),
$$

each corresponding to a traveling wave component of the flow. These eigenfunctions each describe optimal amounts of the streamfunction variance, in descending order of importance, and by definition evolve in the sequence

$$
\begin{aligned}
\operatorname{Re}\left(\Psi_{j}\right) & \rightarrow-\operatorname{Im}\left(\Psi_{j}\right) \rightarrow-\operatorname{Re}\left(\Psi_{j}\right) \rightarrow \operatorname{Im}\left(\Psi_{j}\right) \\
& \rightarrow \operatorname{Re}\left(\Psi_{j}\right),
\end{aligned}
$$

the nature of this evolution being determined by the orthogonal complex principal components $f^{j}(t)$. Of most interest to our case is the first eigenfunction $\Psi_{1}$, which explains the most variance $(40 \%-90 \%$ in the experiments with $A_{F}=2500$ to $5000 \mathrm{~m}$ ).

Use of complex EOF analysis reveals that the un- 

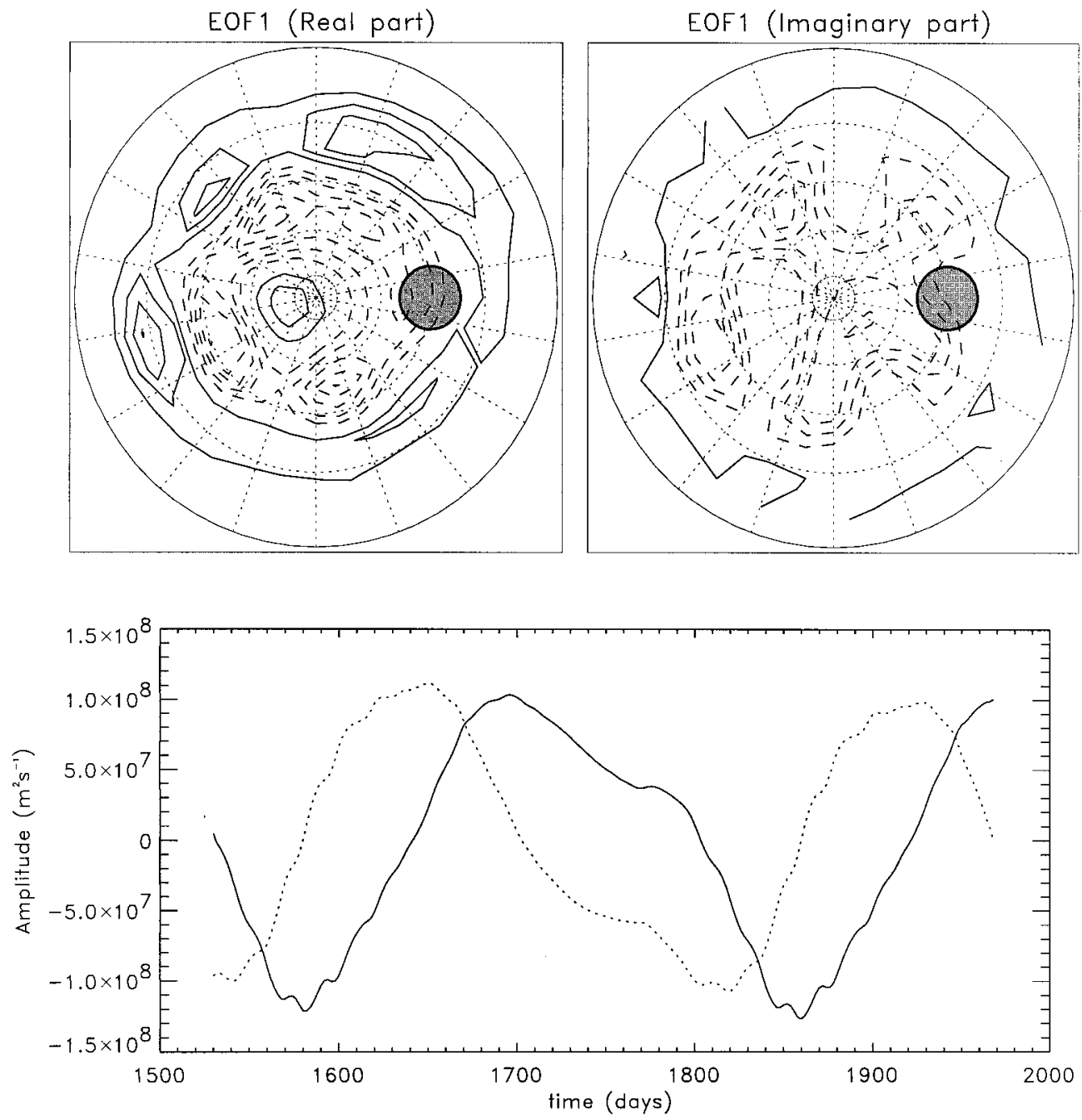

FIG. 7. The structure of the leading complex EOF (left, Re $\psi_{1}$; right, $\operatorname{Im} \psi_{1}$ ) for the $A_{F}=3000 \mathrm{~m}$ experiment as well as its evolution in time. The lower plot shows the time evolution of the complex principal components $\operatorname{Re} f^{1}(t)$ (solid line) and $\operatorname{Im} f^{1}(t)$ (dotted line). The contour interval in the upper-level structural plots is 0.02 .

steady response to localized forcing at large amplitudes falls into two distinct regimes of behavior in our model. For $A_{F}=2500 \mathrm{~m}$ to $A_{F}=3500 \mathrm{~m}$, the flow is observed to oscillate northward and southward, becoming more and then less wavy in the process. This behavior is not zonally localized to the forcing region but affects the whole hemisphere. We refer to this as a global oscillation.

Figure 6 shows the evolution of the Northern Hemisphere PV during one period of the oscillation (from day 1580 to day 1860 ) for the case $A_{F}=3000 \mathrm{~m}$. There is some wave breaking downstream of the mountain and to the south, but in the midlatitudes the waves are characterized by tighter PV gradients, which oscillate but remain wavy. Figure 7 shows the structure and evolution of the first complex EOF $(80.0 \%$ of the total variance) for the streamfunction in this experi- ment. Notice that $\operatorname{Re}\left(\Psi_{1}\right)$ is more zonal while $\operatorname{Im}\left(\Psi_{1}\right)$ is more wavy. With reference to the sequence in Eq. (5), the oscillation can thus be roughly described as follows:

zonal high-latitude flow

$\rightarrow$ wavy low-latitude flow $\rightarrow$ zonal low-latitude flow

$\rightarrow$ wavy high-latitude flow $\rightarrow$ zonal high-latitude flow.

This oscillatory pattern can be seen in the PV field itself (Fig. 6), although some effort is required to detect the difference between the frames.

The period of this global oscillation decreases as the forcing amplitude is increased, from well over 500 days for $A_{F}=2500 \mathrm{~m}$, to 280 days for $A_{F}=3000 \mathrm{~m} \mathrm{(cf.}$ Fig. 7), to 110 days for $A_{F}=3500 \mathrm{~m}$, while the amplitude of the oscillation is similar in each case. 

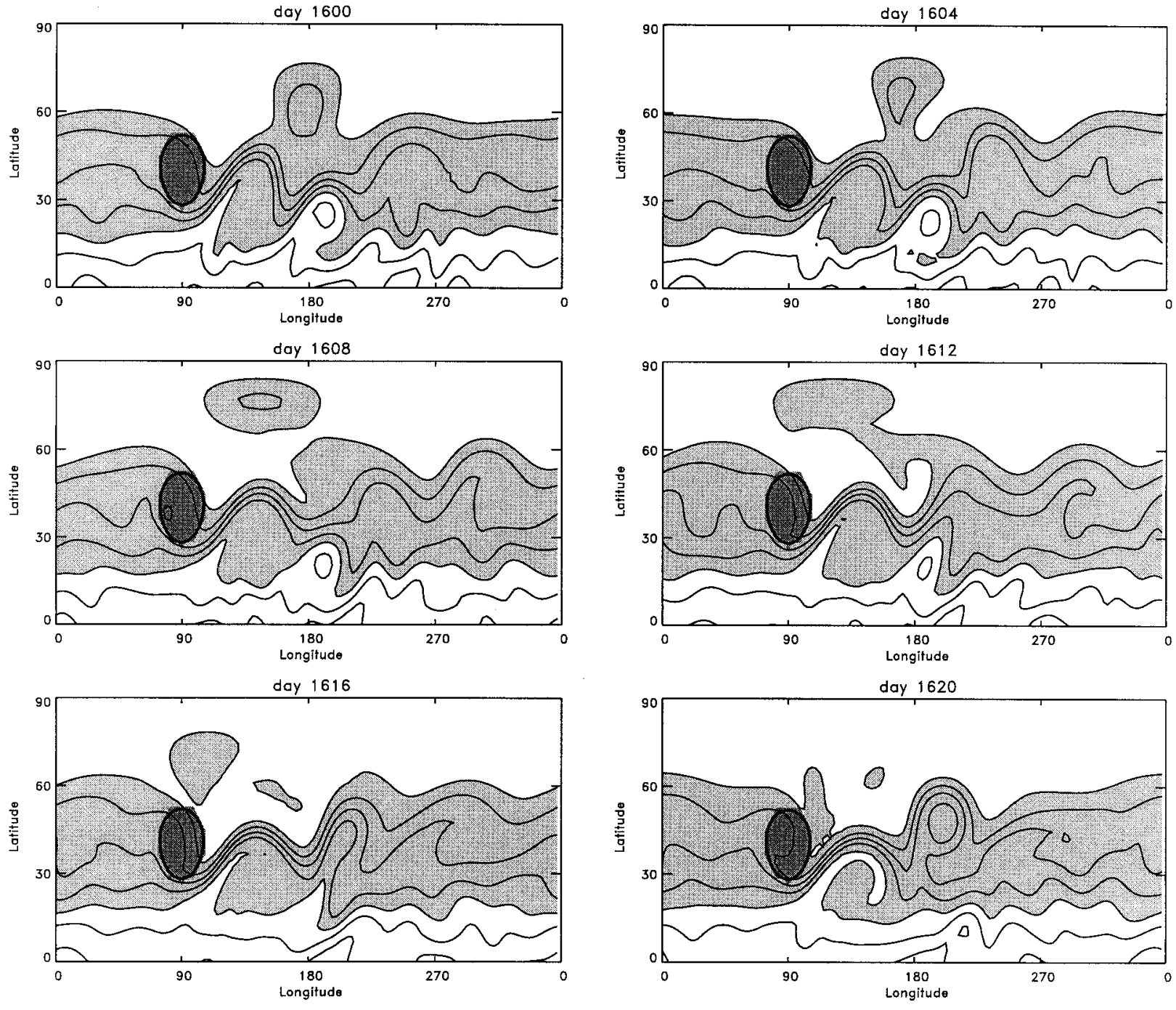

FIG. 8. As in Fig. 6 except showing the Northern Hemisphere PV field during one of the vortex budding events in the $A_{F}=5000 \mathrm{~m}$ experiment.

As the forcing amplitude $A_{F}$ is increased to $4000 \mathrm{~m}$ and higher, the oscillation is observed to enter a different regime. This is characterized by the strong nonzonal jet downstream of the mountain periodically shedding a vortex from its northern flank. We refer to this as a localized oscillation. Figure 8 shows the evolution of the Northern Hemisphere PV field during such a shedding event for the $A_{F}=5000 \mathrm{~m}$ experiment. Figure 9 shows the corresponding evolution of the streamfunction over the same period. The time interval shown is the period 1600-1620 days, by which time the flow has settled into an oscillation with a period of approximately 80 days. At 1600 days, there is a belt of strong PV gradients (collocated with the jet) to the southeast of the mountain and oriented SW-NE. The jet extends to about $\left(50^{\circ} \mathrm{N}, 150^{\circ} \mathrm{E}\right)$ where it splits, the main subtropical branch being at $30^{\circ} \mathrm{N}$ at this time and a weaker branch poleward of $60^{\circ} \mathrm{N}$. Over the course of the next 20 days, the anticyclonic PV anomaly south of the poleward branch is ejected, and the poleward branch collapses, while the subtropical branch rearranges itself such that, by day 1608 , the main jet (the belt of strong PV gradient) is oriented more zonally and extends all the way to $200^{\circ} \mathrm{E}$. Eastward of this longitude the jet is split into two distinct branches. Over the next 12 days the location of this split in the jet is seen to move westward until it is located at $\left(40^{\circ} \mathrm{N}\right.$, $170^{\circ} \mathrm{E}$ ), replacing the split that was destroyed by the ejection of the cyclone. During this time, the cutoff anticyclone at high latitudes drifts westward and dissipates. From 1620 to 1680 days (not shown) the initially strong poleward branch of the jet gradually weakens and the subtropical branch gradually gets stronger until the PV looks very similar to that in the 1600-day snapshot. 

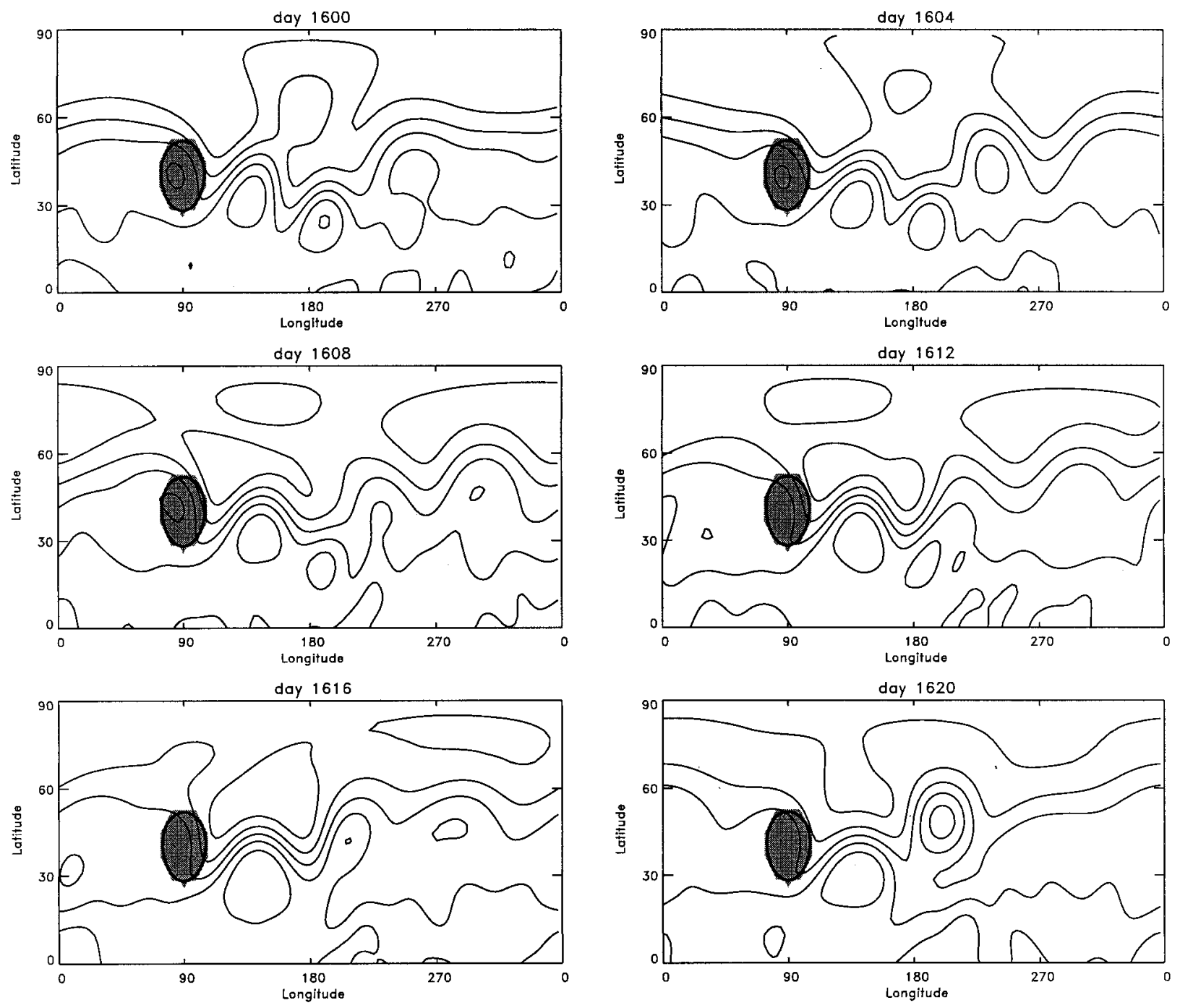

Fig. 9. The evolution of the streamfunction during the vortex budding event shown in Fig. 8 . The contour interval is $1 \times 10^{7} \mathrm{~m}^{2} \mathrm{~s}^{-1}$.

From the complex EOFs perspective, this localized oscillation is illustrated in Fig. 10, which shows the structure and evolution of the first complex EOF of the streamfunction for this experiment $(42.67 \%$ of the total variance). An SWB-type wave train can be observed to propagate from the Tropics to the pole in the jet exit region, with period around 80 days. The propagation is characterized by periods of relatively rapid transition, corresponding to the rapid vortex shedding described above, followed by relatively long periods of gradual change, corresponding to a relaxation of the flow when the subtropical jet becomes stronger and the polar jet weaker. The period of this type of oscillation again varies with the forcing amplitude $A_{F}$. In the 4000-m experiment only two vortex shedding events were recorded in a 500 day period, whereas for 4500 $\mathrm{m}$ the period was around 110 days.

\section{The model sensitivity}

The scenario of the response just presented, that is, the evolution from a steady linear to a steady nonlinear to an unsteady global to an unsteady localized response, is not crucially dependent on the parameter values we have chosen, in the sense that the qualitative transitions of the response are generic. However, the precise values of $A_{F}$ at which the transitions occur are dependent on the dissipative drag $\tau_{D}$. For $\tau_{D}$ smaller than about 30 days, all time variability is suppressed over the range of forcing amplitudes used here (up to $\left.A_{F}=5000 \mathrm{~m}\right)$; similarly, when weaker dissipation was used (values of $\tau_{D}$ greater than 45 days), the timedependent behavior appeared at smaller forcing amplitude. Such dependence of the flow evolution on the drag coefficient has been reported by Huang and Robinson (1995) in a similar model. 

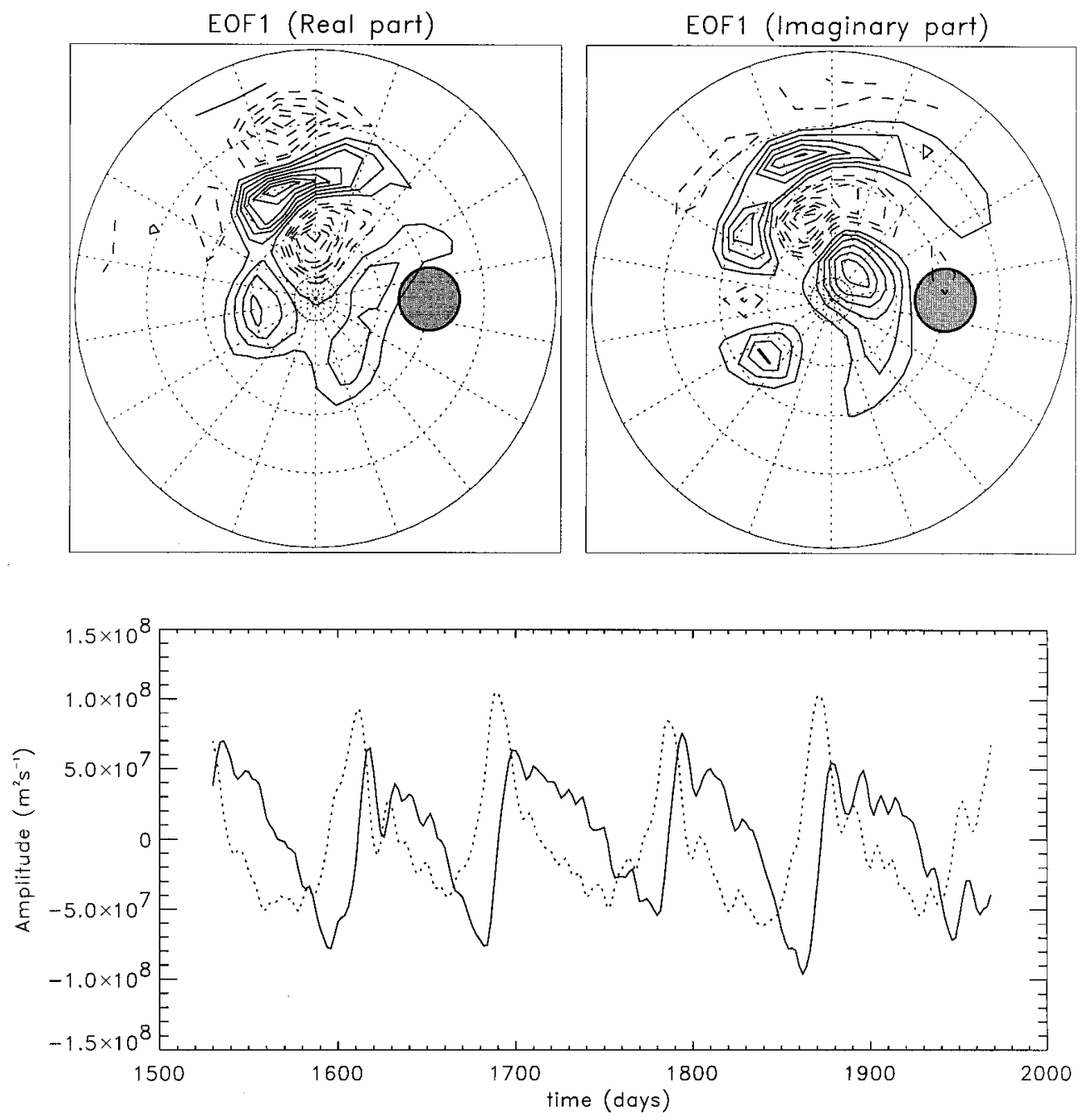

FIG. 10. As in Fig. 7, but for $A_{F}=5000 \mathrm{~m}$.

Another, perhaps more subtle, form of dissipation is present in our model, namely, the hyperdissipation that is introduced via a term of the form $\nu \nabla^{6}$ on the lefthand side of (1). The question of how our results depend on $\nu$ is of course intimately related to how the model response varies as a function of the resolution. We have performed several runs at resolutions of T21, adjusting $\nu$ to $3.6 \times 10^{28} \mathrm{~m}^{6} \mathrm{~s}^{-1}$, the smallest value consistent with numerical stability. As in the experiments of Brunet and Haynes (1996), we discovered a markedly different response. Specifically, we have found that nonlinear time-dependent behavior is never observed at T21 (again, up to $A_{F}=5000 \mathrm{~m}$ ). The flow always becomes steady, even at the largest forcing amplitudes. This is because the smallest value of $\nu$ one must use at T21 has a direct effect on the dynamically important scales of motion. This finding appears consistent with that of Brunet and Haynes (1996), that the wave activity flux into the Tropics is absorbed by the hyperdissipation, leading to spurious stationary be- havior. At T21, the PV redistribution evident in Fig. 8 is overwhelmed by the dissipation. Unsurprisingly, it is also possible to change the regime of behavior observed in the T42 experiments by increasing the value of $\nu$ above that given in Table 1. For example, an experiment with $A_{F}=5000 \mathrm{~m}$ and $\nu=2 \times 10^{27} \mathrm{~m}^{6}$ $\mathrm{s}^{-1}$ resulted in an oscillation of the Northern Hemisphere flow similar to that described above for the $A_{F}$ $=3000 \mathrm{~m}$ experiment.

To use significantly lower values of $\nu$ than those given in Table 1, it is necessary to increase the resolution to T85 in order to maintain numerical stability. Figure 11 shows the evolution of Northern Hemisphere PV during a T85 experiment with $A_{F}=5000 \mathrm{~m}$ and $\nu=5 \times 10^{25} \mathrm{~m}^{6} \mathrm{~s}^{-1}$. This experiment is characterized by a small amplitude periodic oscillation (period $\approx 20$ days) with several important differences, as well as similarities, to the oscillations reported at lower resolution:

- The strong jet downstream of the mountain now ex- 

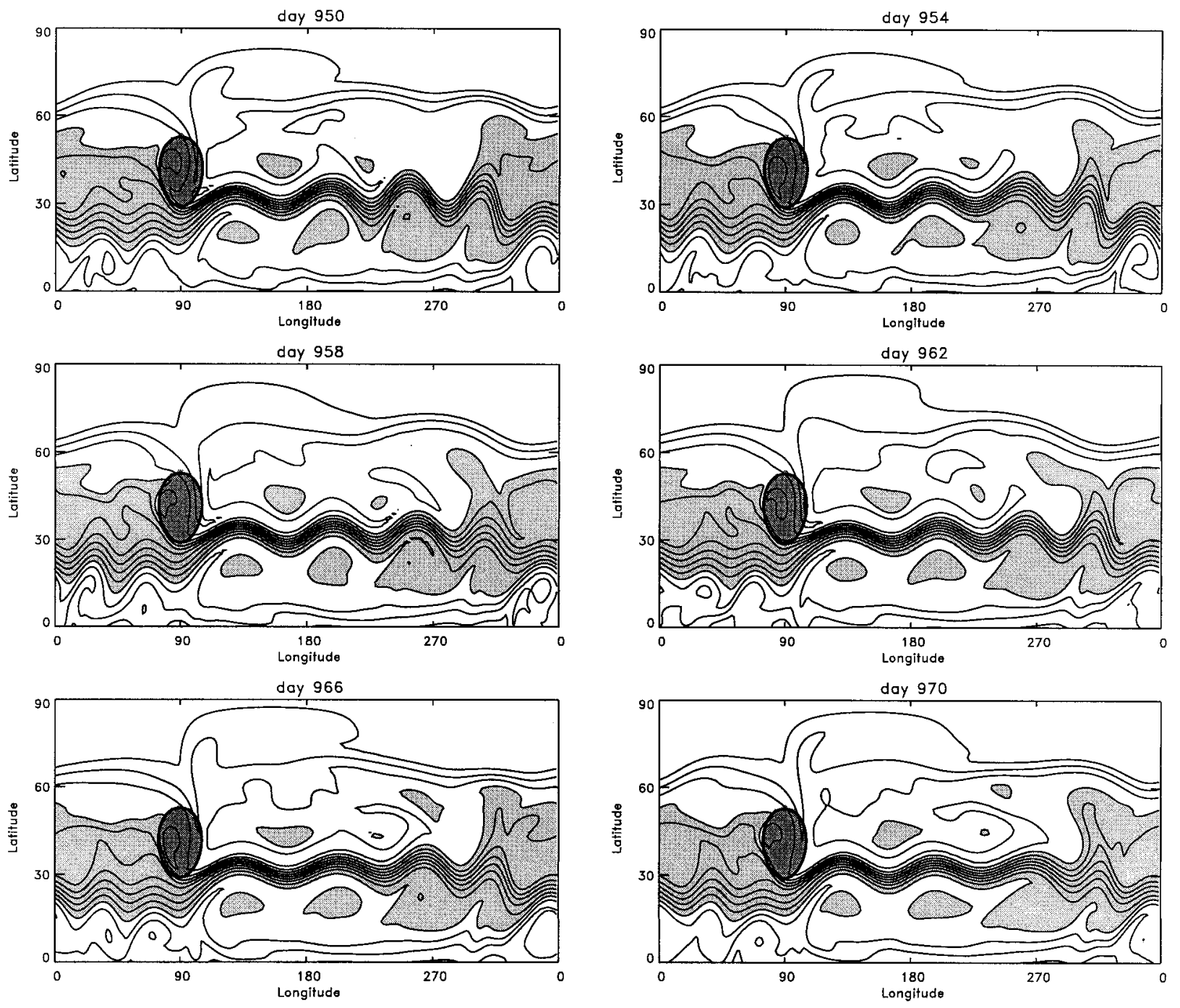

FIG. 11. As in Fig. 6 except showing the Northern Hemisphere PV field during the T85, $\nu=5 \times 10^{25}, A_{F}=5000$ m experiment. The contour interval is $1 \times 10^{-9} \mathrm{~m}^{-1} \mathrm{~s}^{-1}$. Values between $3 \times 10^{-9} \mathrm{~m}^{-1} \mathrm{~s}^{-1}$ and $1 \times 10^{-8} \mathrm{~m}^{-1} \mathrm{~s}^{-1}$ are lightly shaded.

tends halfway around the globe. It is characterized by tight wavy PV gradients surrounded by wellmixed regions.

- Complex EOF analysis shows that the variability of the flow is concentrated almost entirely in the jet exit region, where the flow becomes diffluent (around $250^{\circ}-330^{\circ}$ East longitude).

- The qualitative nature of the oscillation is the same as in the T42 case, involving shedding of vorticity from the north flank of the jet into high latitudes, although the amplitude of the oscillation is much smaller.

This experiment suggests that the time-mean flow is most unstable in regions of diffluence, whereas it is relatively stable in regions of confluence or where it is wavy. It also shows that it is the hyperdiffusion that determines where the jet becomes diffluent and there- fore the region of instability in this model. Other mechanisms may be important in the atmosphere.

Finally, we note in passing another perhaps more surprising sensitivity of the behavior, namely that to the basic zonal flow $\mathbf{v}_{0}$ itself. We have found very high sensitivity to even very small changes in the zonal wind profile used in these integrations. As in the case of varying dissipation, the qualitative behavior was unchanged; nevertheless, the quantitative changes were substantial, even for very similar zonal wind profiles.

\section{Linear stability analysis of the time-averaged flow}

The strong oscillatory time dependence of the flow at large forcing amplitude prompts the question as to how well a linear stability analysis of the time average 

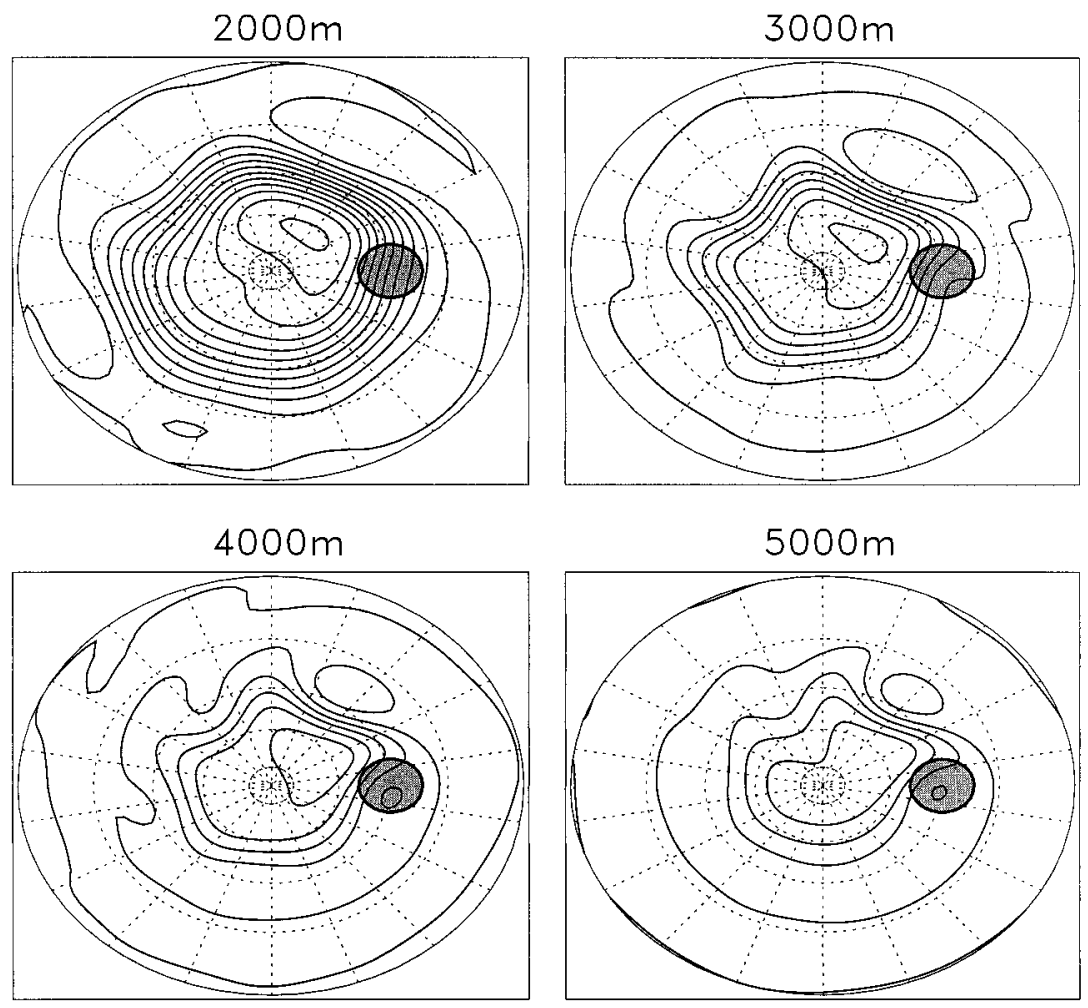

FIG. 12. The time-averaged streamfunction for the four cases $A_{F}=2000 \mathrm{~m}, \ldots, 5000 \mathrm{~m}$. The contour interval is $1 \times 10^{7} \mathrm{~m}^{2} \mathrm{~s}^{-1}$. The dark shaded region indicates the position of the topography.
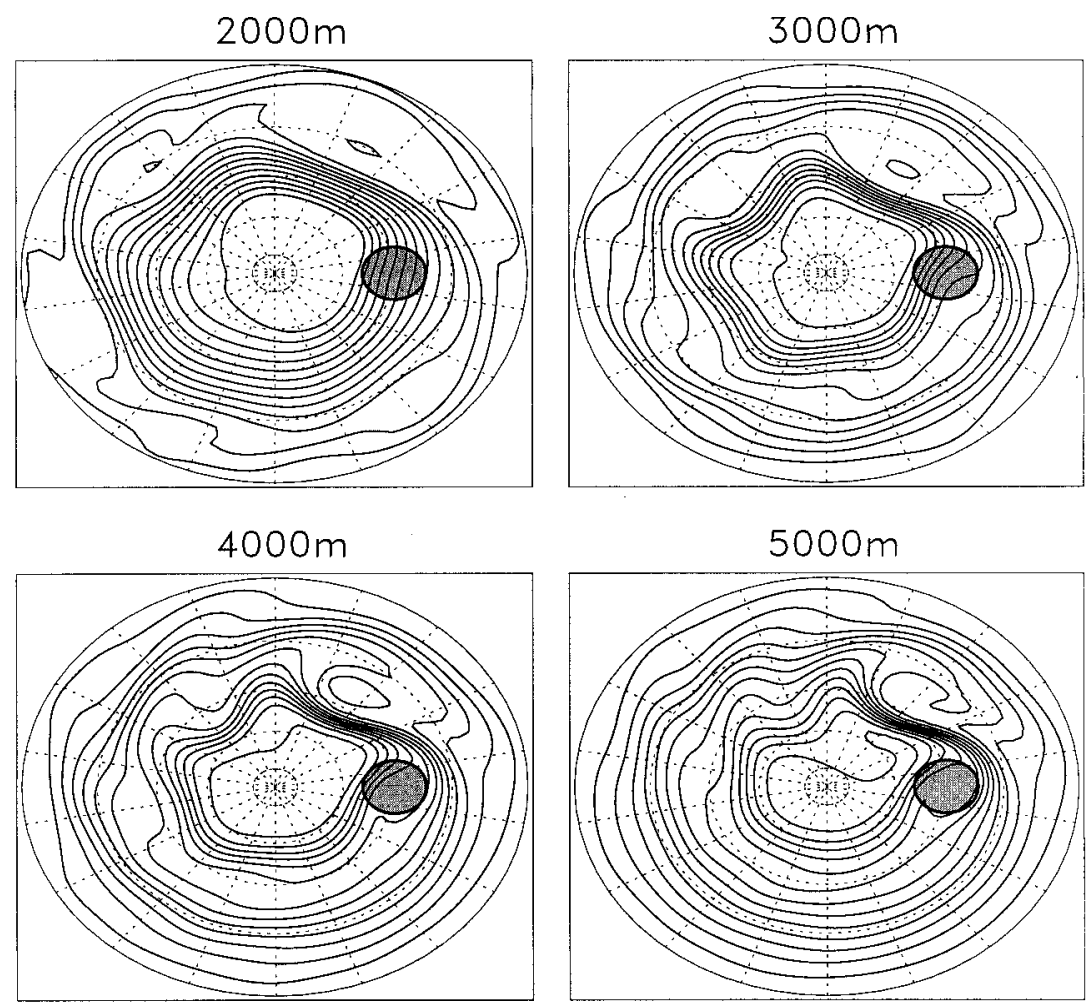

FIG. 13. As in Fig. 12 but showing the time-averaged PV. The contour interval is $1 \times 10^{-9}$ $\mathrm{m}^{-1} \mathrm{~s}^{-1}$. 
of these flows (the procedure used by SWB and others to investigate the characteristics of observed flows) will reproduce the characteristics of the oscillations. We start by constructing the time-averaged states. From the full model runs of 2000 days, we construct time averages from 1500 to 2000 days, to ensure that all initial transients have died out. The time-averaged streamfunction and the corresponding time-averaged PV are shown in Figs. 12 and 13 for the cases $A_{F}=$ $2000 \mathrm{~m}$ to $A_{F}=5000 \mathrm{~m}$. As the amplitude is increased, the time-averaged fields show the formation of a strong jet downstream of the forcing region.

The shallow water equations (1), linearized around each of these time-averaged states, are then integrated for 1000 days, from random initial conditions of very small amplitude. In each case, the solution typically starts growing exponentially after a few days, when the most unstable mode emerges from the initial noise. The properties of these most unstable modes are summarized in Table 2.

The time-averaged model with hyperdiffusivity and drag present (last column of Table 2) becomes unstable for forcing amplitudes greater than $2000 \mathrm{~m}$. Quite strikingly, however, all the unstable modes at large forcing amplitude were found to be stationary (i.e., of infinite period), in contrast with SWB who found many unstable modes with nonzero frequency. The structures of the stationary modes (for the inviscid case) are shown in Fig. 14 as a function of the forcing amplitude $A_{F}$. The modes for $A_{F}=3000-, 4000-$, and 5000-m basic flows have most of their amplitude concentrated in the jet-jet exit region downstream of the mountain, while there is much less concentration of amplitude evident in the $A_{F}=2000 \mathrm{~m}$ case.

From these results it would seem that the linear analysis of the time-averaged flow is able to capture the transition from steadiness to time dependence, in the sense that the time-averaged linear flow becomes linearly unstable at forcing amplitudes comparable to those that mark the transition from steadiness to time dependence in the fully nonlinear model (i.e., $A_{F}=$ $2000 \mathrm{~m}$ ). However, the linear analysis of the timeaveraged flow is unable to predict the amplitude at which the transition from global to local time-dependent behavior occurs. Moreover, since all the most unstable linear eigenmodes at large forcing amplitude are stationary, the linear analysis cannot explain the periods and structures of the oscillations observed in the time-dependent response. This is perhaps not surprising, since the period of the time-dependent response is likely to result from a complex nonlinear equilibration of the growing instabilities.

We have also computed the linear growth rates without hyperdiffusion and with neither hyperdiffusion nor drag (cf. columns 4 and 3 of Table 2). In each case the growth rates increase as the forcing amplitude $A_{F}$ increases (up to $5000 \mathrm{~m}$ ). The linear drag causes the
TABLE 2. The periods and growth rates, in days, of the most unstable modes in the linear experiments.

\begin{tabular}{rcccc}
\hline \hline & & $\begin{array}{c}e \text {-folding } \\
\text { time } \\
\tau_{D}=0 \\
v=0\end{array}$ & $\begin{array}{c}e \text {-folding } \\
\text { time } \\
\tau_{D}^{-1}=45 \mathrm{~d} \\
v=0\end{array}$ & $\begin{array}{c}e \text {-folding } \\
\text { time } \\
\tau_{D}^{-1}=45 \mathrm{~d} \\
v \text { as in } \\
\text { Table } 1\end{array}$ \\
\hline$A_{F}(\mathrm{~m})$ & Period & & & Stable \\
100 & 96 & 1480 & Stable & Stable \\
500 & $\infty$ & 175.4 & Stable & Stable \\
1000 & 64 & 46.2 & Stable & Stable \\
3000 & $\infty$ & 28.8 & 180 & 67.3 \\
4000 & $\infty$ & 21.9 & 40.3 & 54.1 \\
5000 & $\infty$ & 15.2 & 23.2 & 30.7 \\
\hline
\end{tabular}

growth rate to be reduced ${ }^{2}$ by about $\tau_{D}$, and the hyperdiffusion stabilizes the system further.

An interesting side issue is the behavior of the linear model in the limit of very small forcing amplitude and in the absence of drag and hyperdiffusivity. As can be seen from Table 2, we find that, perhaps surprisingly, inviscid growth rates remain positive (though very small) at forcing amplitudes so small that the nonzonality of the basic flow is barely perceptible (cf. Fig. 4). Moreover, the most unstable normal modes in the small-amplitude inviscid case possess a time-periodic global spatial structure, as opposed to the modes at large forcing amplitude which, as already mentioned, are all localized and stationary.

As an example, we show in Fig. 15 half the period of the oscillation for the linearly most unstable disturbance in the case $A_{F}=100 \mathrm{~m}$, for which the PV of the steady response is nearly indistinguishable from zonal. This structure, with half a period of about 48 days, can be described quite well as the superposition of two Rossby-Haurwitz waves. Such structures are typical of the instability of a Rossby-Haurwitz wave (e.g., Baines 1976). It is, however, characteristic of that instability that there is an amplitude threshold that must be exceeded. In the small amplitude limit, instability occurs through resonant triads (Gill 1974). Exactly resonant triads cannot usually be found on the sphere, and so the basic-state wave amplitude must be large enough for the wave-wave interaction to overcome dispersion of the growing waves (Baines 1976). That we do not find a threshold (in the amplitude range investigated) may result from the fact that the basic state we use, with barely detectable yet localized nonzonality, can be thought of as a superposition of many Rossby-Haurwitz waves, thus increasing the availability of almost resonant triads.

\footnotetext{
${ }^{2}$ SWB show that the same linear drag applied in the barotropic vorticity equations reduces the growth rate of all modes by exactly $\tau_{D}$ (see their appendix A).
} 

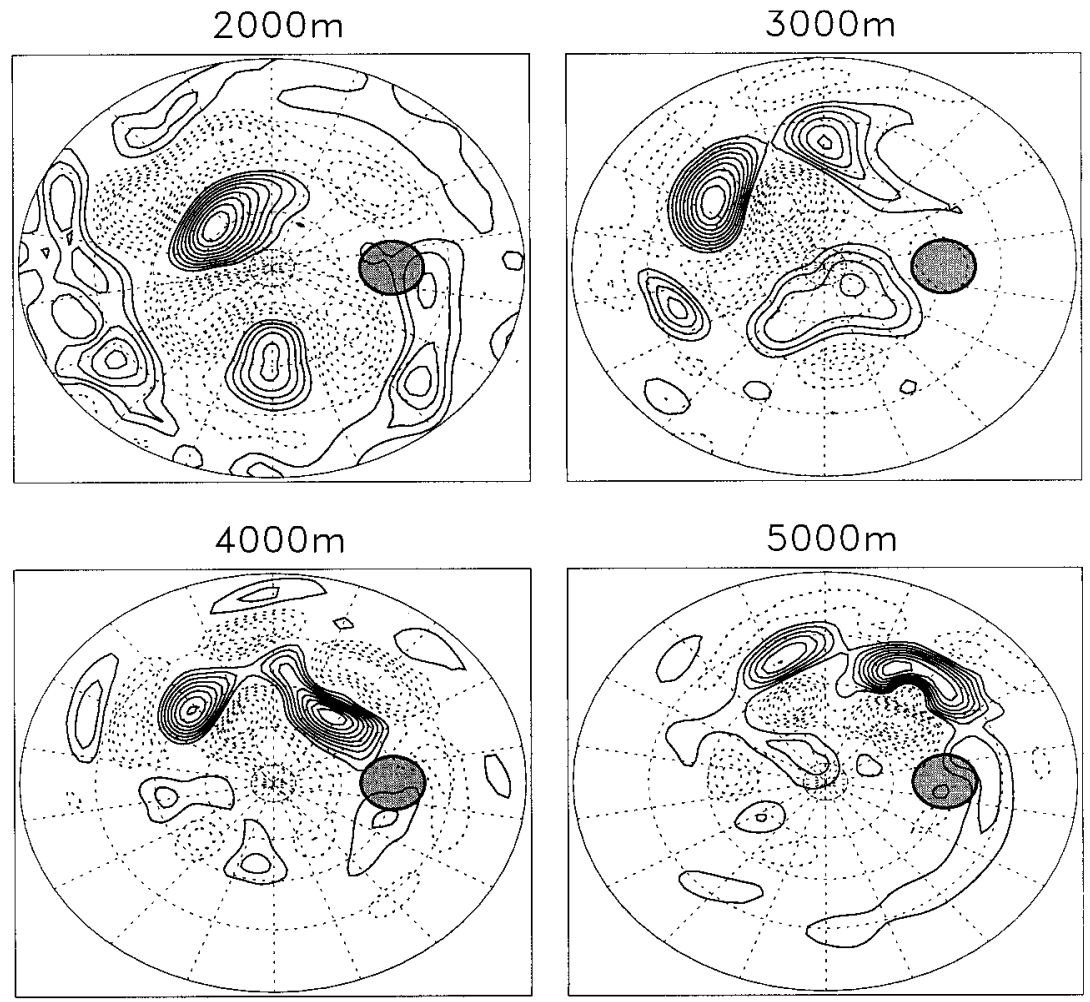

FIG. 14. The spatial structure of the streamfunction of the linearly most unstable modes calculated from the time-averaged flows for the four cases $A_{F}=2000 \mathrm{~m}, \ldots, 5000 \mathrm{~m}$. All the modes are stationary. The contour interval is arbitrary.

\section{Discussion}

These experiments have shown how forcing a simple westerly flow with a single localized mountain can reproduce one of the observed characteristics of observed quasi-stationary waves, namely, low-frequency variability localized in the jet exit regions. Moreover, it was found that the amplitude of the topographic forcing needs to exceed a certain threshold before an oscillation of the entire Northern Hemisphere flow yields to a more "realistic" localized oscillation.

How directly the results of our model can be applied to the real atmosphere is not entirely clear. Our results are in agreement with the conclusions of Borges and Sardeshmukh (1995) (see also Sardeshmukh et al. 1997) in that oscillatory behavior is found only for unrealistically large wave amplitudes and/or unrealistically weak dissipation. Even for $\tau_{D}=45$ days, a drag time much longer than is realistic for deep tropospheric systems, oscillatory behavior only sets in when the time-averaged flow has exaggerated wave features (cf. Fig. 12 with $A_{F} \geq 3000 \mathrm{~m}$ and compare with typical monthly mean upper-tropospheric flows in northern winter). With dissipation that is significantly more rapid (though still weaker than is realistic), the oscillatory behavior disappears altogether. Thus, while the similarity between the patterns of observed low- frequency variability in the atmosphere and of the variability found in this and similar models continues to be intriguing, many discrepancies remain.

One interesting conclusion may be drawn from this study, however. Having constructed a model in which the time variability is controllable (in this case via the forcing amplitude $A_{F}$ ), we can attempt to answer the following important question: given a complex, evolving, time-dependent flow, what can one learn from a linear analysis of the time-averaged flow? In our simple model, the linear analysis is unable to capture both the periodicity and the spatial structures of the fully evolving flow. Whether these results generalize to more complex and hopefully more realistic models remains an open question. It does however suggest the possibility that linear analysis may be an inadequate tool for understanding atmospheric time variability.

Finally, we remark that our model, which in many ways is as simple as can be conceived, was found to be highly sensitive both to the choice of basic zonal wind and drag coefficient, as well as to the numerical resolution used. We were surprised to find such sensitivity, since it has rarely been reported in numerical studies of atmospheric flows. We suggest, therefore, that much caution may be necessary when using numerical models for direct quantitative comparison with observations. 

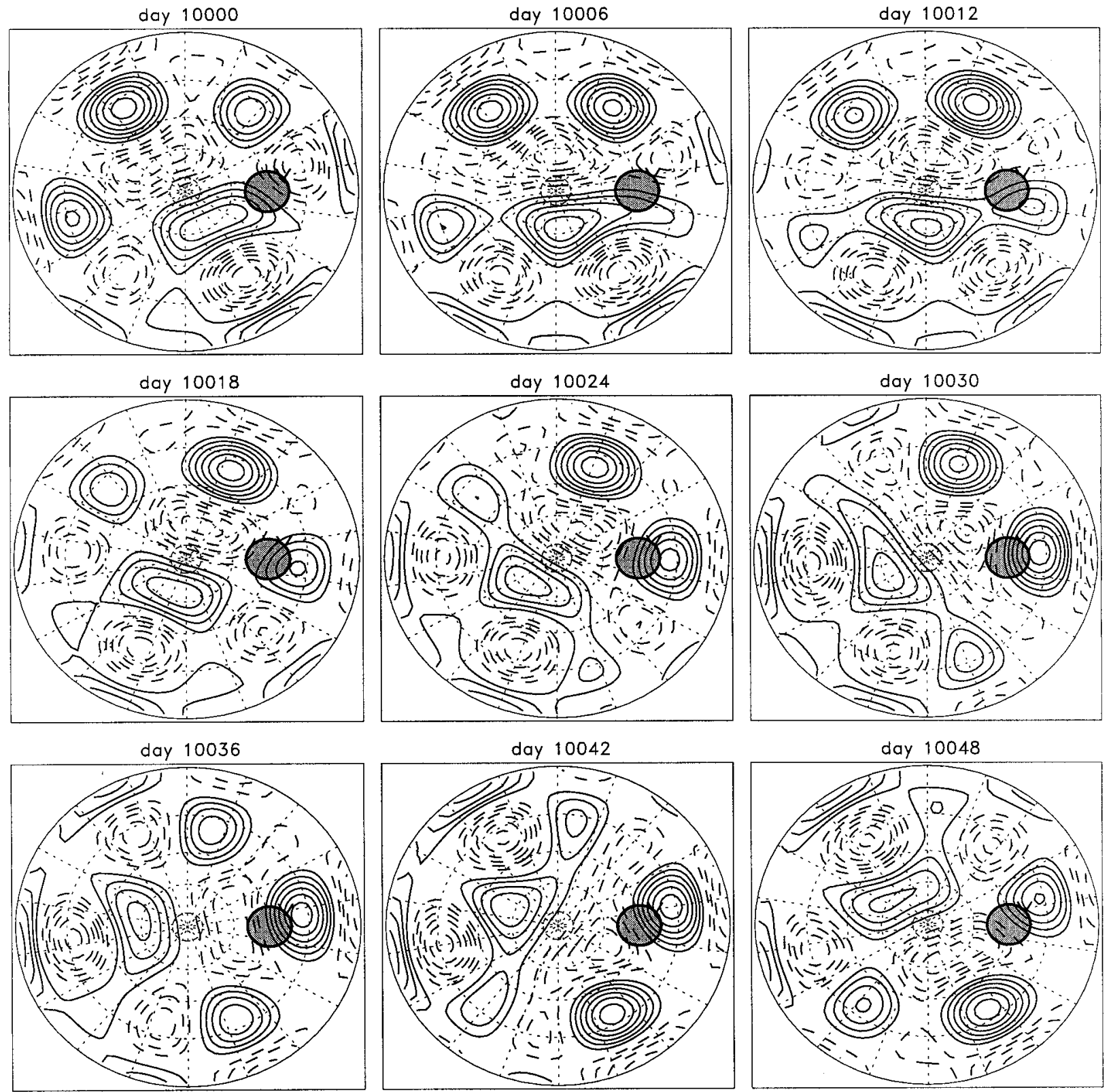

FIG. 15. One half cycle of the spatial evolution of the linear streamfunction of the most unstable mode for the near-zonal $A_{F}=100 \mathrm{~m}$ case.

Acknowledgments. We are grateful to Grant Branstator, Isaac Held, and Darryn Waugh for several useful discussions. The computations presented here were performed at the Pittsburgh SuperComputing Center. This work was supported by the National Science Foundation under grants to MIT and Columbia University.

\section{REFERENCES}

Andrews, D. G., 1984: On the stability of forced nonzonal flows. Quart. J. Roy. Meteor. Soc., 110, 657-662.
Baines, P. G., 1976: The stability of planetary waves on a sphere. J. Fluid Mech., 73, 193-213.

Borges, M. D., and P. D. Sardeshmukh, 1995: Barotropic Rossby wave dynamics of zonally varying upper-level flows during northern winter. J. Atmos. Sci., 52, 3779-3796.

Branstator, G. W., and I. M. Held, 1995: Westward propagating normal modes in the presence of stationary background waves. J. Atmos. Sci., 52, 247-262.

Brunet, G., and P. H. Haynes, 1996: Low-latitude reflection of Rossby wave trains. J. Atmos. Sci., 53, 482-496.

Frederiksen, J. S., and P. J. Webster, 1988: Alternative theories of atmospheric teleconnections and low-frequency fluctuations. Rev. Geophys., 26, 459-494.

Gill, A. E., 1974: The stability of planetary waves. Geophys. Fluid Dyn., 6, 29-47. 
Grose, W. L., and B. J. Hoskins, 1979: On the influence of orography on large-scale atmospheric flow. J. Atmos. Sci., 36, 223-234.

Hack, J. J., and R. Jakob, 1992: Description of a shallow water model based on the spectral transform method. NCAR Tech. Note NCAR/TN-343+STR, 39 pp. [Available from NCAR, P.O. Box 3000, Boulder, CO 80307.]

Horel, J. D., 1984: Complex principal component analysis: Theory and examples. J. Climate Appl. Meteor., 23, 1660-1673.

Huang, H. P., and W. A. Robinson, 1995: Barotropic model simulations of the North Pacific retrograde disturbances. J. Atmos. Sci., 52, 1630-1641.

Sardeshmukh, P. D., M. Newman, and M. D. Borges, 1997: Free barotropic Rossby wave dynamics of the wintertime low-frequency flow. J. Atmos. Sci., 54, 5-23.

Simmons, A. J., J. M. Wallace, and G. W. Branstator, 1983: Barotropic wave propagation and instability, and atmospheric teleconnection patterns. J. Atmos. Sci., 40, 1363-1392.

Waugh, D. W., L. M. Polvani, and R. A. Plumb, 1994: Nonlinear, barotropic response to a localized topographic forcing: Formation of a "tropical surf zone" and its effect on interhemispheric propagation. J. Atmos. Sci., 51, 1401-1416.

Webster, P. J., and J. R. Holton, 1982: Cross-equatorial response to middle-latitude forcing in a zonally varying basic state. $J$. Atmos. Sci., 39, 722-733. 